\title{
Finite element analysis of a rupture-induced deformation of a carotid atherosclerotic plaque with intraplaque hemorrhage
}

\author{
Hamed ESMAEILI MONIR*, Omi SENJU**, Toshiyasu OGATA******, Tooru INOUE**, \\ Noriyuki SAKATA**** and Hiroshi YAMADA* \\ *Dept. of Biological Functions Engineering, Graduate School of Life Science and Systems Engineering, Kyushu Institute of Technology \\ 2-4 Hibikino, Wakamatsu-ku, Kitakyushu 808-0196, Japan \\ E-mail:yamada@life.kyutech.ac.jp \\ ** Dept. of Neurosurgery, Faculty of Medicine, Fukuoka University \\ 7-45-1 Nanakuma, Jonan-ku, Fukuoka 814-0180, Japan \\ ***Dept. of Neurology, Faculty of Medicine, Fukuoka University \\ ****General Medical Research Center, Faculty of Medicine, Fukuoka University
}

Received: 28 August 2017; Revised: 20 October 2017; Accepted: 27 November 2017

\begin{abstract}
Intraplaque hemorrhage (IPH), bleeding in a plaque, is caused by a neocapillary rupture in an atherosclerotic plaque. We used contrast-enhanced ultrasonography to diagnose carotid atherosclerotic plaques before carotid endarterectomy (CEA), a surgical operation to remove an arterial intimal layer including a plaque lesion. We found lumenward (inward) deformation in some cases of ruptured plaques with IPH. The aim of this study was to evaluate the mechanical effects of infiltrated blood in the lipid core on the luminal shape of the ruptured plaque in the short-axis view. We created a finite element model of a carotid artery bifurcation with a ruptured plaque based on a sample obtained from CEA. As physiological loads, we assigned pressures on the surfaces of the lumen and the lipid core, the sum of a gradual pressure drop in the artery obtained from computational fluid dynamics analysis and a uniform pressure, and a constant longitudinal stretch. In the simulation, the fibrous cap in the ruptured model became almost flat in the short-axis view with lumenward deformation, being less deformed than that observed in ultrasonography. The simulation results show that inward deformation of the fibrous cap is correlated with an equal pressure in the lumen and the lipid core. In comparison, a hyperelastic model of soft unruptured plaque reproduced a round lumen. A better understanding of contrast-enhanced ultrasonography images from a mechanical perspective may facilitate the morphological identification of plaque rupture with IPH.
\end{abstract}

Keywords : Vascular mechanics, Finite element method, Atheromatous plaque, Intraplaque hemorrhage, Rupture, Carotid endarterectomy, Contrast-enhanced ultrasonography

\section{Introduction}

The rupture of atheromatous plaques in carotid arteries is a cause of cerebrovascular events (Hennerici, 2004). Among the determinants of risk of a stroke are degree of stenosis and plaque surface morphology (European Carotid Surgery Trialists' Collaborative Group, 1991; Lovett et al., 2004). Intraplaque hemorrhage (IPH), bleeding in a plaque, is caused by a neocapillary rupture in an atherosclerotic plaque (Pasterkamp and van der Steen, 2012). We used contrast-enhanced ultrasonography to identify a rupture in carotid atherosclerotic plaques with IPH before carotid endarterectomy (CEA), a surgical operation to remove an arterial intimal layer including a plaque, and in some cases found lumenward deformation of ruptured plaques with IPH (Hamada et al., 2016). The plaque surface morphology on carotid angiography, classified as ulcerated, irregular, or smooth, is a highly sensitive marker of plaque instability 
(Rothwell et al., 2000). However, few studies have focused on the correlation between the luminal shape of the plaque and the mechanical conditions (Yamada and Sakata, 2013).

In recent years, various imaging modalities, including ultrasonography, angiography, magnetic resonance imaging (MRI), and computer tomography (CT), have been developed to examine the risk of plaque rupture. In T1-weighted MRI images of unruptured carotid plaques, soft plaques that are composed of a large lipid necrotic core and/or IPH are detected as regions with high signal intensities (Takano et al., 2012).

The luminal surfaces of plaques are usually inflated outward in the short-axis view (Saam et al., 2005). Yamada and Sakata (2013) conducted a finite element (FE) analysis, demonstrating a round lumen and a low level of pressure $(<2 \mathrm{kPa})$ in the liquid-like lipid core of an atheromatous carotid artery under physiological loadings with intraluminal pressure of $16 \mathrm{kPa}$, for example. In contrast, a lumenward bulge or a partially straight line has been observed for fibrous caps with IPH in short-axis MRI images (Cai et al., 2005; Chu et al., 2004; Saam et al., 2005). In addition, using contrast-enhanced ultrasonography, a promising modality for visualizing ulceration in atherosclerotic arteries (Hamada et al., 2016), we identified clinical cases in which a ruptured human carotid atherosclerotic plaque was accompanied by lumenward deformation. However, the cause of such morphological changes has yet to be revealed.

In this study, we investigated carotid artery bifurcations (CAB) with ruptured and unruptured plaques using FE modeling methods. We used cross-sections of the intimal layer obtained from CEA to develop a geometrical model of a ruptured plaque. We supplemented the cross sections with ultrasonographic images of the CAB, taken prior to CEA, and a lateral computer tomography (CT) image of the head and neck. To examine the plaque's distinctive surface morphology, we compared FE analysis results of ruptured and unruptured plaque surface models with in-vivo ultrasonography observations. The morphology was expected to be caused by pressure elevation in the lipid core from blood infiltration through a hole in the plaque.

\section{Methods}

\subsection{Clinical cases of carotid endarterectomy}

We registered a total of 28 patients who underwent contrast-enhanced ultrasonography before CEA at Fukuoka University Hospital between January 2013 and December 2013. CEA was performed as described elsewhere (Ogata et al., 2014; Hamada et al., 2016). We made morphological observations of the carotid artery using short- and long-axis-view ultrasonographic images. In total, 4 of 28 patients exhibited lumenward bulged plaques with rupture. In three of the four cases, we identified plaque rupture and hemorrhage from histological observations. We selected the plaque of a 74-year-old man with 70\% carotid artery stenosis for FE modeling of the CAB [see Fig. 1(a), (b)].

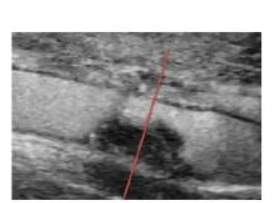

(a)

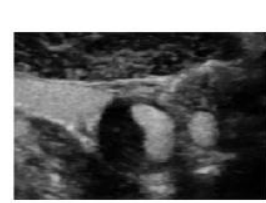

(b)

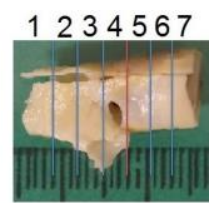

(C)

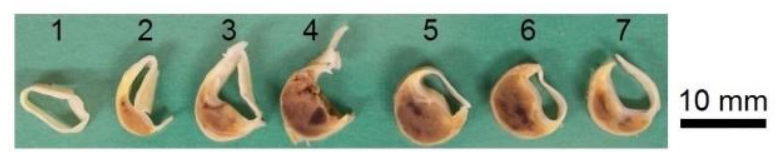

(d)

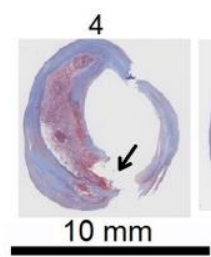
5
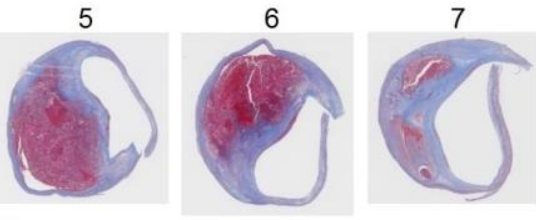

(e)

Fig. 1 (a) Long-axis in vivo ultrasonographic image of the carotid artery. (b) Short-axis ultrasonographic image of segment No. 5. (c) Formalin-fixed intimal layer extracted by carotid endarterectomy. (d) Formalin-fixed cross-sections. The top surface is the right (distal) side for segments No. 1-5 and the left (proximal) side for No. 6 and 7. Thus, segments No. 5 and 6 show the same cut surface. (e) Masson trichrome staining of segments. The arrow in segment 4 indicates the location of the plaque rupture. The scale bar is the same for (a) to (d). 
The tissue sample was fixed in $10 \%$ formalin in $0.1 \mathrm{~N}$ phosphate buffer ( $\mathrm{pH} 7.4)$ within $24 \mathrm{~h}$ [see Fig. 1(c)] and cut circumferentially into segments with intervals of approximately $3 \mathrm{~mm}$ in the longitudinal direction [see Fig. 1(c) and (d)]. Microscopic sections $(3 \mu \mathrm{m})$ were cut from the paraffin-embedded tissue samples and subjected to hematoxylin and eosin, elastic van Gieson, and Masson trichrome staining. We identified IPH in the lipid core under light microscopy [see Fig. 1(e)]. This study was approved by the Research Ethics Committee of the University Hospital, Fukuoka University (No. 11-10-03) and informed consent was obtained from each patient.

\subsection{Finite element modeling}

We created an FE model of the $\mathrm{CAB}$ with a ruptured plaque ('ruptured model') and an unruptured plaque ('unruptured model') to investigate the mechanical effect on the morphology of the ruptured plaque. The features of these models are summarized in Table 1 and the details are described below.

Table 1 Ruptured and unruptured models.

\begin{tabular}{c|c|c|c}
\hline \hline & Ruptured model & Unruptured soft plaque model & Unruptured hard plaque model \\
\hline Geometry & Fig. 3 (a) left panel & Fig. 5(a) left panel & Fig. 5(a) left panel \\
Vascular wall & Hyperelastic (polynomial & Hyperelastic (polynomial & Hyperelastic (polynomial \\
& form), Eqs. (1) and (3) & form), Eqs. (1) and (3) & form), Eqs. (1) and (3) \\
Lipid core & Cavity connected to vascular & Hyperelastic (neo-Hookean), & Same material as vascular \\
lumen through a hole & Eqs. (2) and (4), no hole & wall, no hole \\
Longitudinal stretch & 1.1 & 1.1 & 1.1 \\
Pressure on surface & Vascular lumen and lipid core & Vascular lumen & Vascular lumen \\
& cavity (15-16 kPa) & $(15-16 \mathrm{kPa})$ & $(15-16 \mathrm{kPa})$ \\
\hline
\end{tabular}

\subsubsection{Geometrical modeling}

We developed a three-dimensional (3D) geometric model of the intimal dissected layer of CAB using Rhinoceros computer-aided design (CAD) software (ver. 4.0; Robert McNeel and Associates, Seattle, WA), importing photographs of segment Nos. 1 - 5 and 7 in Fig. 1(d). As shown in Fig. 2(a), we extracted available parts of the profile from the segments (indicated in black). We estimated unavailable parts of the profile (indicated in red), making it round in shape with the same luminal circumference for distorted parts or assuming some parts that had been lost due to plaque rupture or CEA. We aligned these profiles so that the blue lines in Fig. 2(a), which were determined to be located almost in the middle of the plaque and fibrous cap, were positioned on the same medial plane.

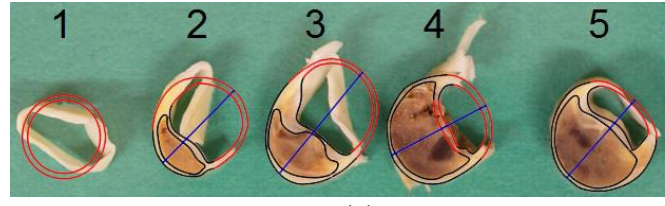

(a)

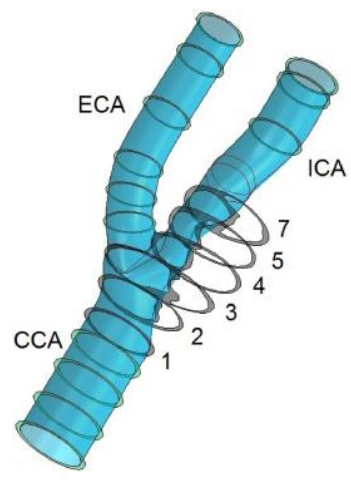

(b)

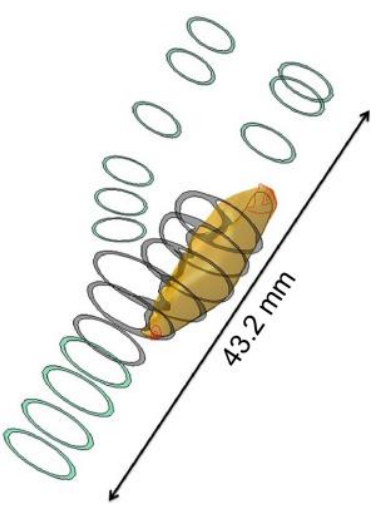

Fig. 2 (a) The profiles used to create the lumen, lipid core, and outer surface of the dissected intimal region. Note that segment No. 6 was not used [see the caption in Fig. 1(d)]. (b) Left panel: lumen surface (in blue), cross-sections 1-5 and 7 (in gray) and additional circular profiles (in green). Right panel: surface of the lipid core (in yellow), additional closed curves on the surface of the lipid core (in red).

In the CAD software, we added extra circular profiles of normal intimal regions to the common carotid artery (CCA) and the internal carotid artery (ICA) to ensure a sufficient length for computational fluid dynamics (CFD) analysis. The lipid core region was extracted from the CEA sample and the cross-section was assumed to reduce 
gradually, from the proximal end in the additional length of $0.8 \mathrm{~mm}$, and from the distal end in the additional length of $4.7 \mathrm{~mm}$ [see Fig. 2(b)]. The intimal wall at segment No. 1, a hollow circular region, was extended by $12.2 \mathrm{~mm}$ from the proximal side of the CCA in the proximal direction. The bifurcation was placed between segments 3 and 4 , as identified from the CEA sample.

Circular profiles in the short axis were added from the bifurcation of the intimal model, for the normal intimal region of the external carotid artery (ECA), along a longitudinal length of $43.2 \mathrm{~mm}$. In a lateral view on a 3D CT image of the head and neck, the internal diameters were almost uniform in the CCA ( $>1 \mathrm{~cm}$ proximal from the bifurcation), ICA $(>0.5 \mathrm{~cm}$ distal from the bifurcation, and ECA $(\approx 1 \mathrm{~cm}$ distal from the bifurcation $)$. We then obtained ratios of the uniform diameters for the above ICA and ECA regions, under loading conditions, of 0.78 and 0.7 , respectively, with respect to that for the CCA. In segment No. 1, the internal diameter was calculated from the luminal circumference of $6.03 \mathrm{~mm}$. Using the above ratios, the uniform diameters of the ICA and ECA at the distal region of the model were estimated as $4.7 \mathrm{~mm}$ and $4.2 \mathrm{~mm}$, respectively.

The outer wall of the intima was expanded radially for the CCA and ICA, such that their thicknesses of the whole wall took the assumed values of 1.17 and $0.86 \mathrm{~mm}$ in the normal wall regions, respectively (Sommer et al., 2010). A hollow circular wall was added to the intima of ECA with the same thickness as the normal region of ICA, merging its outer surface to those of ICA and CCA. Finally, a 3D geometrical model was created for the vascular wall with a lipid core using these surfaces.

A 1.55-mm-wide rupture in the fibrous cap [see histology of segment No. 4 in Fig. 1(e)] was modeled by subtracting a cylinder $1.55 \mathrm{~mm}$ in diameter from the fibrous cap region in the FE model, resulting in a connection of the lumen to the lipid core region. The unruptured model had no holes in the fibrous cap.

Figure 3 shows the ruptured model. The 3D geometrical models were imported to Abaqus (ver. 6.14; SIMULIA, Providence, RI, USA) and meshed with 733840 4-node linear tetrahedral elements with linear pressure (C3D4H). Figure 3(a) presents a cross-section of the geometrical model and the FE model of the CAB with a ruptured plaque. The lumen and the lipid core were modeled as a cavity to investigate the effect of pressure (see Table 1 and Section 2.2.3). Figure 3(b) is a cross-sectional geometry and meshed CFD model. Figure 3(c) shows that there was a change in the profile of the fibrous cap through application of the loft command in Rhinoceros CAD software to create surfaces of the lumen and lipid core by fitting a smooth surface through selected profile curves. For the unruptured case, the lipid core was modeled as hyperelastic material to examine the effect of its stiffness [see Table 1 and the left-hand panel in Fig. 5(a)].

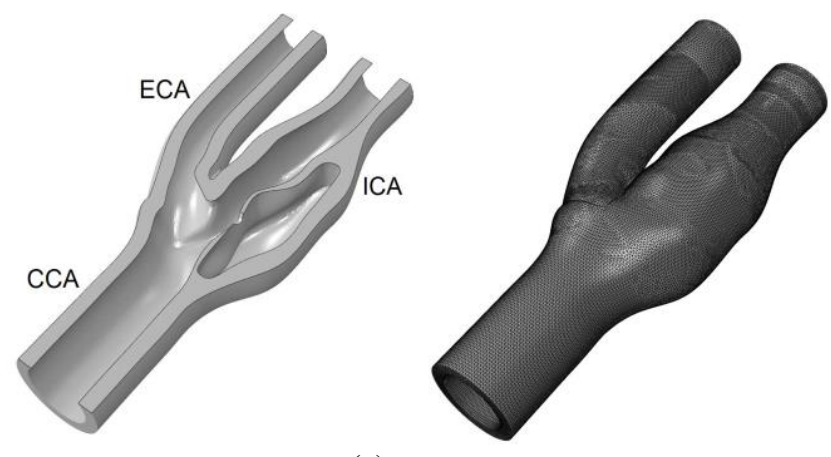

(a)

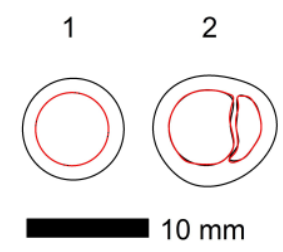

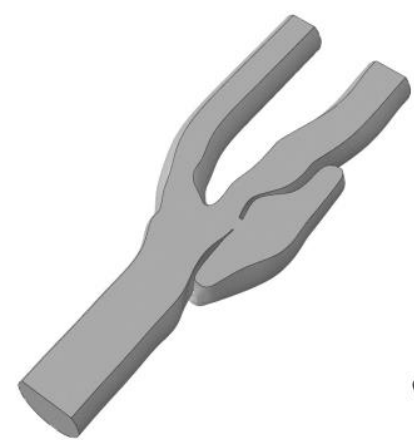

(b)

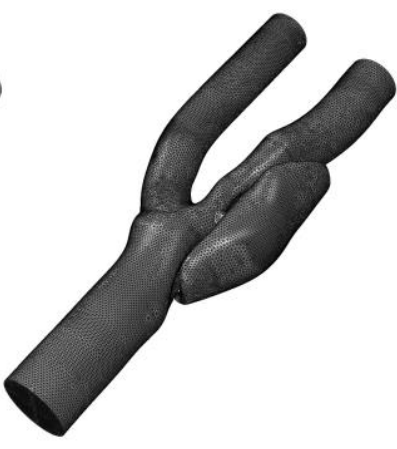

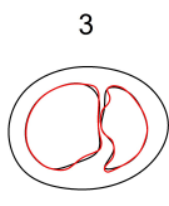

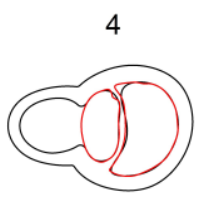

(c)

Fig. 3 Ruptured model. (a) Geometrical model in the ruptured case (left) and its corresponding FE model (right). (b) Geometry of the lumen, lipid core, and the cylindrical pathway between the lumen and the lipid core (left) and their corresponding CFD model (right). (c) Profiles of the fibrous cap before and after applying the loft command in Rhinoceros CAD software (before loft: red; after loft: black). 


\subsubsection{Constitutive equations}

We assumed incompressible, isotropic, and homogeneous hyperelastic materials for the arterial wall and lipid core (Yamada and Sakata, 2013). Then we postulated a strain-energy density function in a reduced polynomial form for the arterial wall as

$$
W=\sum_{i=1}^{5} C_{i}\left(I_{1}-3\right)^{i}+\frac{H}{2}\left(J^{2}-1\right)
$$

and a strain-energy density function of neo-Hookean material for the lipid core in the unruptured model with a soft plaque as

$$
W=C_{10}\left(I_{1}-3\right)+\frac{H}{2}\left(J^{2}-1\right)
$$

where $I_{1}$ is the first invariant of the right Cauchy-Green deformation tensor, $H$ is an indeterminate pressure, and $J$ is the determinant of deformation gradient tensor. The material constants $C_{i}(i=1,2, \ldots, 5)$ and $C_{10}$ were given as (Yamada et al., 2010; Yamada and Sakata, 2013)

$$
\begin{aligned}
& C_{1}=2.16(\mathrm{kPa}), C_{2}=3.16(\mathrm{kPa}), C_{3}=232(\mathrm{kPa}), C_{4}=2.78(\mathrm{kPa}), C_{5}=3.07(\mathrm{kPa}) \\
& C_{10}=0.01(\mathrm{kPa}) .
\end{aligned}
$$

\subsubsection{Boundary conditions}

A CFD model of the lumen connected with a lipid core cavity [see Fig. 3(b)] was created with 642617 4-node tetrahedral fluid elements (FC3D4) in Abaqus (ver. 6.14) to estimate the pressure distribution along the longitudinal axis and in the lipid core region, which was to be used as a boundary condition for the ruptured model.

Atherosclerosis causes stenosis, which can make the flow turbulent $(\mathrm{Ku}, 1997)$. The flow patterns in moderately and severely stenosed carotid arteries have been simulated by transitional turbulence flow models (Tan et al., 2008). In this study, we chose the RNG $k-\varepsilon$ turbulence model (Yakhot et al., 1992), a two-equation turbulence model for turbulence kinetic energy $k$ and turbulence energy dissipation rate $\varepsilon$ (see Appendix). We used a blood density of 1050 $\mathrm{kg} / \mathrm{m}^{3}$ for $\rho$ and dynamic viscosity of $3.5 \times 10^{-3} \mathrm{~Pa} \cdot \mathrm{s}$ for $\mu$. We also took the same reference pressure at the distal ends of the ECA and ICA. As an inlet velocity condition, a steady and uniform velocity of $0.588 \mathrm{~m} / \mathrm{s}$ was applied at the proximal side of the CCA (see Appendix A2).

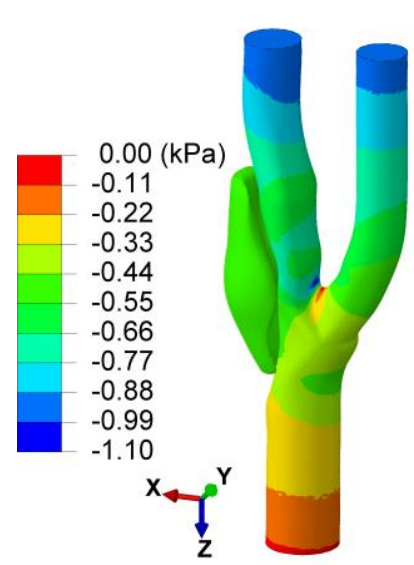

(a)

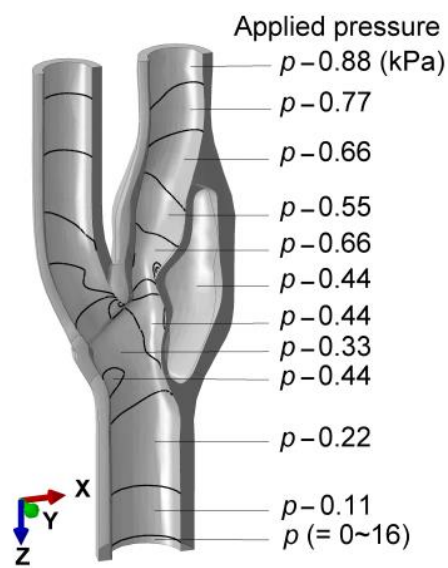

z

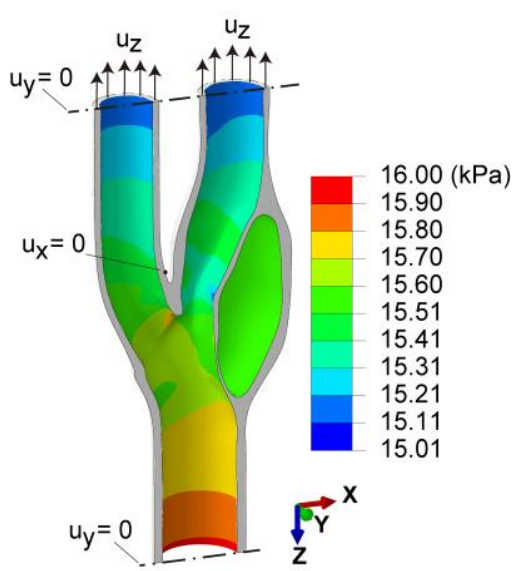

(b)

Fig. 4 (a) Pressure distribution on the surfaces of the lumen and lipid core cavity derived from CFD analysis with the inlet pressure condition of $0 \mathrm{kPa}$ (left). Representative image showing the pressure regions created on the inner surface of the FE models, based on the pressure drop derived from the CFD analysis, and how the pressure was applied to them (right). (b) Boundary conditions applied to the model for a systolic pressure of $16 \mathrm{kPa}$ at the proximal side of the common carotid artery. A variable $u_{i}$ denotes a displacement in $i$ axis.

In the ruptured FE model, pressures were applied on the surfaces of the lumen wall and the lipid core cavity. The CFD analysis reproduced the pressure distribution on the surface of the lumen and lipid core cavity, as shown in the left panel of Fig. 4(a). We divided the pressure drop between the inlet and outlet into 10 levels with intervals of $0.11 \mathrm{kPa}$ due to the technical difficulty of applying pressure with a continuous change. Then, we mapped them on the wall 
surfaces of the lumen and lipid core cavity in the ruptured model. The uniform blood pressure was added to the above pressure distribution so that the pressure at the proximal end of the CCA ranged from 11-16 $\mathrm{kPa}$ (diastolic and systolic pressures) over a cardiac cycle. Figure 4(b) shows the distribution of surface pressure in a case in which the inlet pressure is $16 \mathrm{kPa}$. The pressure on the outer surface of the artery was always kept at zero.

In the ruptured model, we applied a longitudinal stretch of $10 \%$ (Delfino et al., 1997), which stabilizes the arterial wall morphologically (Jackson et al., 2005), by assigning displacement $u_{z}$ to the distal end surfaces of the ICA and ECA and fixing the proximal end surface of CCA, as shown in Fig. 4(b). We also applied an out-of-plane displacement constraint, $u_{y}=0$, in the proximal and distal lines [dot-dashed lines in Fig. 4(b)] and $u_{x}=0$ at a selected point on the outer wall [a dot in Fig. 4(b)] along the same long-axis plane. The same boundary conditions of pressures on the lumen wall and longitudinal stretch were applied to the unruptured FE models.

\section{Results}

\subsection{Mechanical state of the plaque under physiological loading}

Figure 5 shows long-axis (upper panels) and short-axis views (lower panels) of the geometry in the unloaded state (left panel) and the maximum principal stress in the physiologically loaded state with an inlet pressure of $16 \mathrm{kPa}$ and longitudinal stretch of $10 \%$ (central and right panels), for the unruptured model with a soft plaque [Fig. 5(a)] and for the ruptured model [Fig. 5(b)]. In the unruptured model with a soft plaque, the fibrous cap bulged outward [see the outward $\operatorname{arcs}$ A and B in Fig. 5(a)]. In the ruptured model, the cavity of the lipid core increased in size [compare the regions marked with an asterisk in the long- and short-axis views of the second column between Fig. 5(a) and Fig. 5(b)] and the fibrous cap deformed lumenward in the short-axis planes [compare the horizontal position of the fibrous cap with respect to the plaque shoulders in the second column panels between Fig. 5(a) and Fig. 5(b)] under physiological loading. The maximum principal stress was largest (>500 kPa) in the fibrous cap near the rupture hole, which is the thinnest region (approximately $60 \mu \mathrm{m}$ ), for the ruptured model or the corresponding region for the unruptured model (see locations $\mathrm{C}$ and D). We confirmed that the results for uniform pressure of $16 \mathrm{kPa}$ were quite similar to the ones we obtained in the present study.

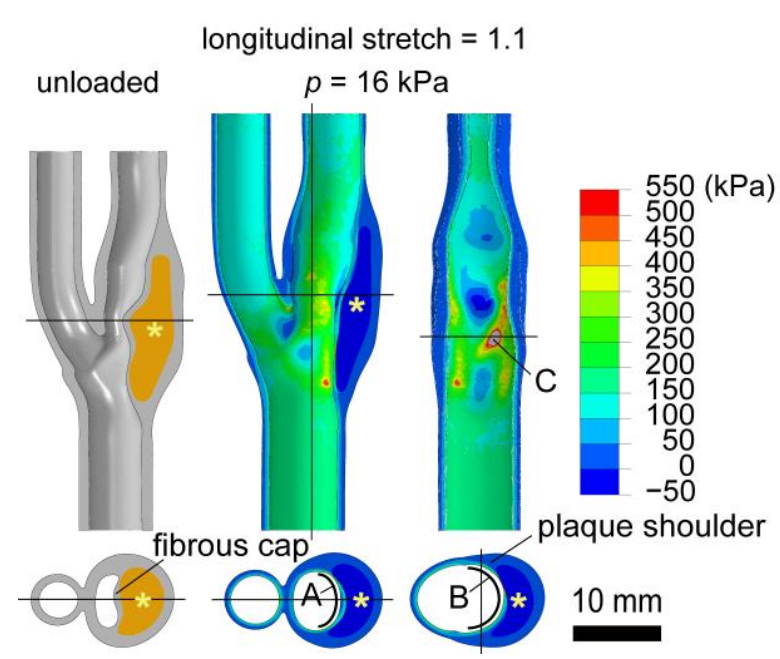

(a)

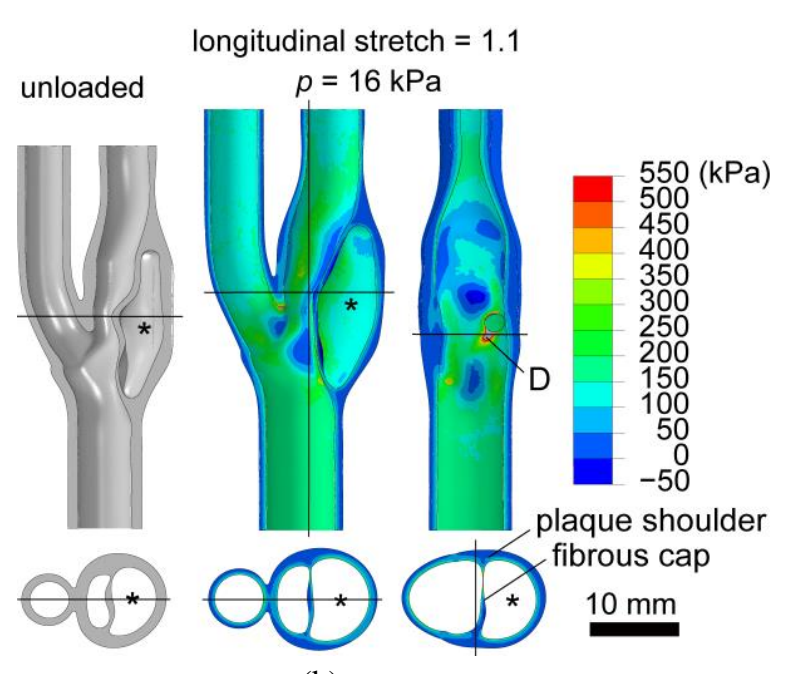

(b)

Fig. 5 Long-axis and short-axis views of the geometry in an unloaded state and maximum principal stress in a physiologically loaded state with an inlet pressure of $16 \mathrm{kPa}$ and longitudinal stretch of $10 \%$ for the (a) unruptured model with a soft plaque and (b) ruptured model. The horizontal lines in the upper panels indicate identical cross-sections and show the locations of the short-axis view (left and central panels: most stenotic; right panel: thinnest region of the fibrous cap near the rupture hole). The vertical line in the central panel shows the long-axis plane to view the right panel. The horizontal line in the right panel passes just below the rupture hole, which is where the fibrous cap is thinnest. The lines in the lower panels show the view planes for the upper panels. Asterisks indicate the lipid core, A and B the curves of the fibrous cap, and C and D the locations of highest stress.

\subsection{Short-axis profiles of plaques compared between ultrasonographic and FE analyses}

Figure 6(a) compares the short-axis profiles of ruptured plaque in the FE model with ultrasonographic images at 
diastole and systole. The fibrous cap in the FE model was deformed lumenward, but did not bulge as much as in the ultrasonographic image. Figure 6(b) shows a round lumen in an unruptured plaque on both ultrasonography and Masson trichrome staining, which agrees qualitatively with the deformed shape of the unruptured model with a soft plaque shown in Fig. 5(b).

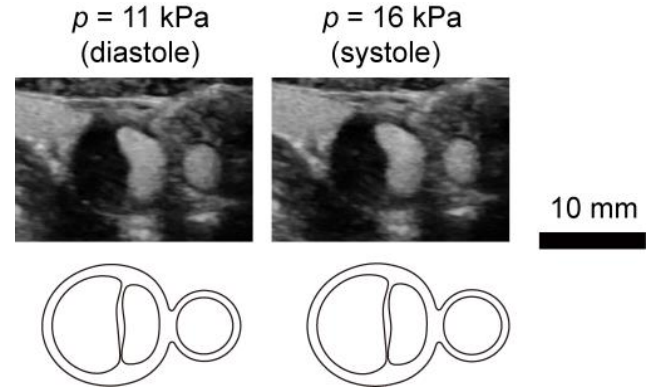

(a)

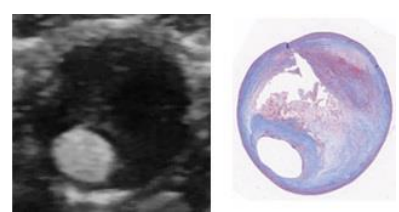

(b)

Fig. 6 (a) Comparison of ultrasonographic images in a short-axis view of the carotid artery at diastole and systole with the corresponding profiles of ruptured FE models at inlet pressures of 11 and $16 \mathrm{kPa}$ in the same short-axis plane of Fig. 5. (b) A short-axis view of a carotid artery with an unruptured plaque demonstrating a round shape of the lumen: ultrasonography image (left) and Masson trichrome staining image (right).

\section{Discussion}

We created an FE model of a CAB, for a ruptured plaque with IPH, based on a CEA sample and simulated the deformation behavior under physiological loading conditions. We evaluated the effect of the pressure elevation in the lipid core due to plaque rupture on the lumenward deformation of the plaque. In the ruptured model, the equivalent levels of pressure in the lumen and the lipid core caused lumenward deformation of the fibrous cap, but this was less pronounced than that observed by ultrasonography. We also created an unruptured model with soft hyperelastic material for the lipid core. The unruptured model had a round lumen surface with an outward bulge due to the intraluminal pressure, which was much higher than the pressure in the lipid core.

\subsection{Variation of the cross-sectional geometry of the fibrous cap in the FE analysis}

In this study, four cases had a lumenward bulge in ultrasonography; IPH was confirmed in three cases based on microscopic observations and the other case had calcification in the plaque region based on ultrasonography.
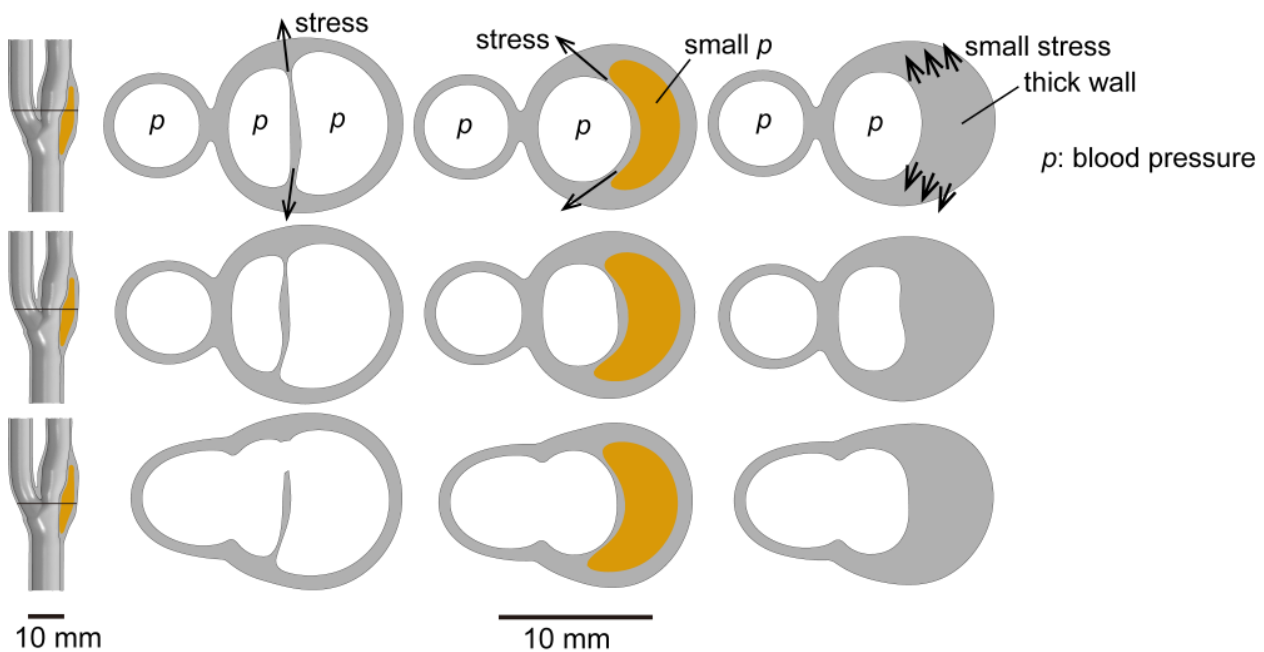

$10 \mathrm{~mm}$

Fig. 7 Variation in the deformed shape of transverse cross sections of the ruptured model (second column), unruptured model with a soft lipid core [Eq. (4)] (third column), and unruptured model with a hard plaque that was assumed to be as stiff as the vascular wall [Eq. (3)] (fourth column) at a longitudinal interval of $2 \mathrm{~mm}$. The left column shows the vertical cross-section in the deformed state. Yellow regions indicate the soft lipid core in the unruptured model. Stress and pressure are illustrated by arrows and the letter $p$, respectively, in the top row panels. 
A plaque with a soft lipid core has a membrane-like fibrous cap, but one with a hard lipid core tends to have a thick and stiff wall. Figure 7 shows the variation in the geometry of the fibrous cap in transverse cross-sections at three different longitudinal locations separated at an interval of $2 \mathrm{~mm}$ in the bifurcation for the ruptured model, the unruptured model with a soft lipid core [Eq. (4)], and the unruptured model with a hard plaque, which was assumed to be as stiff as the vascular wall [Eq. (3)]. The curvature of the luminal surface of the fibrous cap varied due to its non-uniform thickness.

The fibrous cap in the ruptured model deformed lumenward at all locations. In the unruptured model with a soft lipid core, the curvature along the boundary of the lipid core was always convex outward, with its pressure significantly lower than the luminal blood pressure (Yamada and Sakata, 2013). Compared with the unruptured soft plaque model (see the third column in Fig. 7), the unruptured hard plaque model, which had no IPH, deformed to a small degree (see the fourth column in Fig. 7) because much more energy is required to stretch the plaque region circumferentially. A lumenward bulge of the lumen surface was plausible not only in the case of IPH with rupture but also in the case of a hard unruptured plaque.

Using FE analysis with the surface pressures and longitudinal stretches of $0 \%, 10 \%$, and $20 \%$, we also confirmed that the longitudinal stretch elongates the plaque longitudinally and reduces its circumference. The variation in cross-sectional geometry is therefore not due to longitudinal stretch.

\subsection{Geometry of the fibrous cap in the plaques with IPH}

Figure 8 presents the three clinical cases of carotid atherosclerotic plaques that ruptured and hemorrhaged. These were categorized histologically according to the classification of the American Heart Association (AHA) (Stary et al., 1995). Ultrasonography revealed a lumenward bulge with an ulcer. Case \#3 was used for the FE analysis in this study. Note that the cross-sections in the sample photo, ultrasonography, and MRI are not identical in each case. The ultrasonography and MRI in case \#1 near the bifurcation and cases \#2 and \#3 demonstrated that the fibrous cap bulged toward the lumen, while the ultrasonography at a distal location in case \#1 demonstrated an almost straight fibrous cap at a distal location.

To check the sensitivity of the FE model to changes in the luminal profile, we carried out FE analyses for 0.1 mm-thick disc-like FE models with profiles of segments No. 5 and No. 7 (see Fig. 2 (c) and Fig. 9), before and after executing the loft command in Rhinoceros CAD software. We compared the arterial profiles in short-axis view of the arterial models in the unloaded and loaded states for the two disc-like models, as well as for the present 3D FE model (see Fig. 10). For each segment, the fibrous cap showed a similar flat shape among the models under physiological loading conditions. These observations indicated that the deformed shape of the membrane-like fibrous cap is not sensitive to the shape change in the undeformed state following execution of the loft command.

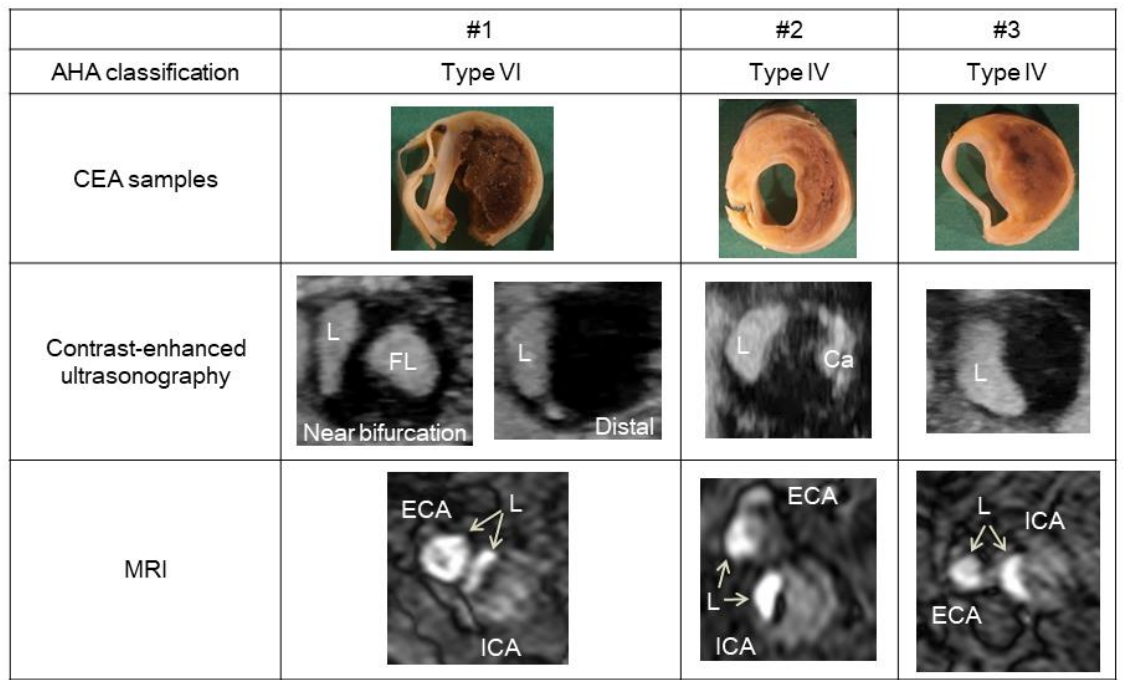

Fig. 8 Three clinical cases of carotid atherosclerotic plaques. Ultrasonography revealed a lumenward bulge with an ulcer and the histological observations identified hemorrhage and rupture. Case \#3 was used for the FE analysis in this study. Ca, calcified; ECA, external carotid artery; FL, false lumen; ICA, internal carotid artery; L, lumen. Note that the cross-sections in the sample photo, ultrasonography, and MRI are not identical in each case. 
Atheromatous plaque rupture may allow blood to infiltrate into the lipid core and increase its volume (Davies, 1994). Plaque rupture accompanied by IPH thus elevates the pressure in the lipid core. According to the balance of force on the membrane (Laplace equation), the pressure difference between the two sides of a membrane mediates its curvature; an equal pressure led to the flat surface of the membrane or fibrous cap in this study. At almost the same pressure in the lumen and the lipid core, namely, $15.6 \mathrm{kPa}$ in the lipid core and $15.1 \mathrm{kPa}$ at the most stenotic location on the lumen surface (see Fig. 4), the fibrous cap deforms outward in the soft plaque model (see the third column in Fig. 7) and it moves lumenward with its surface almost flat in the ruptured model (see the second column in Fig. 7).

Regarding plaques with IPH, the fibrous cap may rupture if it has a lumenward bulge or a partially flat surface \{Fig. 2 (Chu et al., 2004); second row in Fig. 1 (Saam et al., 2005); Fig. 3 (Cai et al., 2005); third column of baseline images in Fig. 1 (Groen et al., 2007)\} in the short-axis view. In comparison, some reports have described outwardly bulging plaques with/without IPH \{without IPH: Fig. 1 (Cai et al., 2005) and right CCA of patient 2 in Fig. 1 (Saam et al., 2013); with IPH: left ICA of patient 1 in Fig. 1 (Saam et al., 2013)\}, which is consistent with the result of the unruptured FE model without hemorrhage with the obtained pressure of $<2 \mathrm{kPa}$ in the lipid core.

For plaques with IPH, one may ask whether the pressure in the lipid core cavity increases due to plaque rupture or whether plaque rupture is induced by the increased pressure in the lipid core. If the pressure in the lipid core increased to the level of lumen blood pressure, the thin fibrous cap would become almost straight, as recognized from the balance of force on the membrane or the FE analysis (Yamada and Sakata, 2013). In our studies and the literature, there has been no such demonstration for unruptured plaques. This suggests that the rupture comes first and results in a lumenward bulge of plaques with IPH.

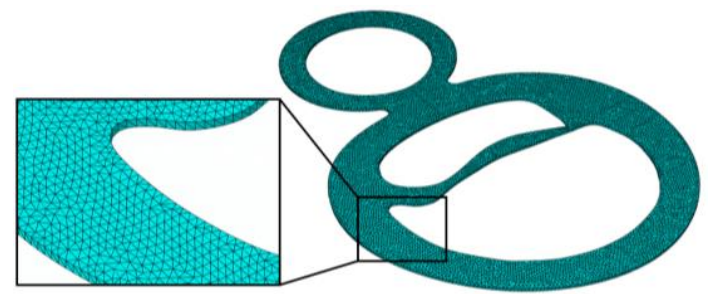

Fig. 9 An example of the 0.1-mm-thick disc-like FE model (segment No. 5 with the geometry before applying the loft command).

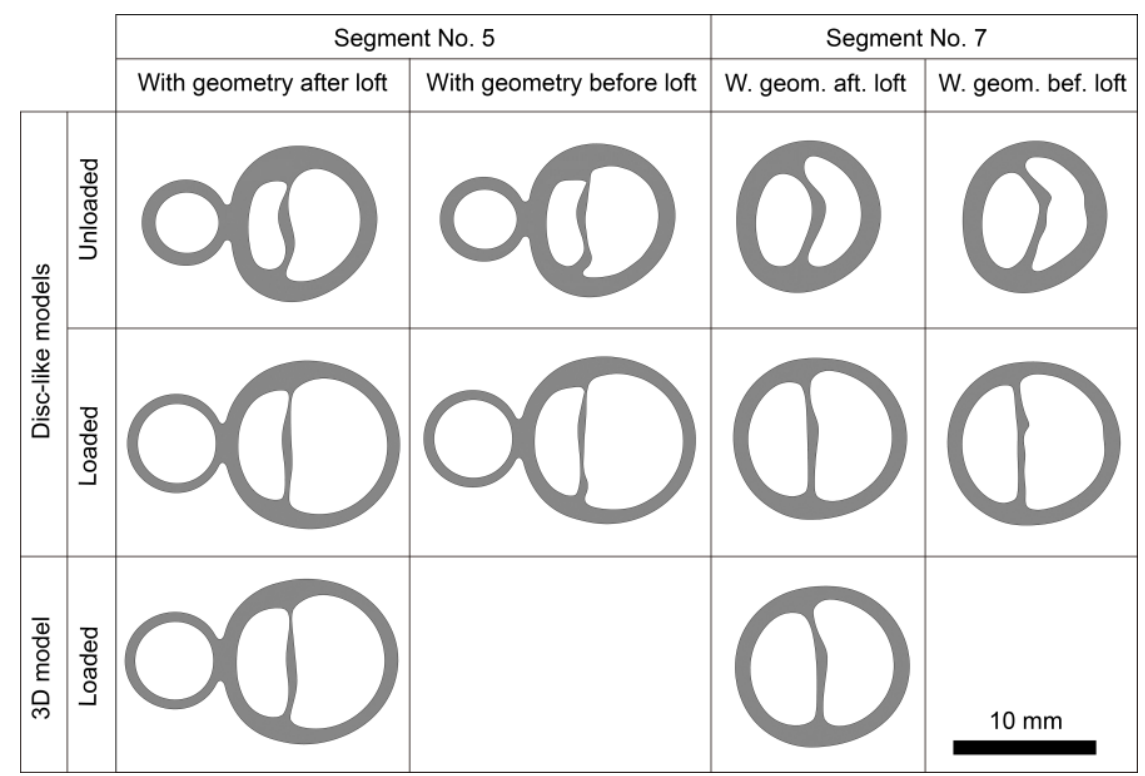

Fig. 10 Arterial profiles in the short-axis view of the unloaded and loaded states for the 0.1-mm-thick disc-like FE models of segments No. 5 and 7: profiles before and after executing the loft command in Rhinoceros CAD software [see Fig. 3(c)] and the present 3D FE models.

\subsection{Stress concentration in the fibrous caps}

The stress distributions of both ruptured and unruptured plaque models show that stress was concentrated at $>500$ 
$\mathrm{kPa}$ in the thinnest region of the fibrous cap near the hole of rupture. We confirmed that stress was concentrated in the same region for a very stiff fibrous cap using a disc-like FE model. The level of stress is higher than the commonly used critical stress of $300 \mathrm{kPa}$ for rupture, and the cap thickness is similar to the threshold thickness of $65 \mu \mathrm{m}$, which is prone to rupture (Cardoso and Weinbaum, 2014; Holzapfel et al., 2014). Teng et al. (2010b) also pointed out that the stresses at the sites of both minimum thickness in the fibrous cap and maximum curvature in the plaque shoulder should be evaluated as candidates of plaque rupture.

\subsection{Blood flow, velocity, pressure drop, and fluid shear stress in the carotid artery}

The flow rate in the $68 \%$ stenotic ICA in our CFD model was $431 \mathrm{~mL} / \mathrm{min}$. This was comparable to the mean flow rate measured invasively as $390 \pm 91 \mathrm{~mL} / \mathrm{min}$ [mean \pm standard deviation (SD)] for 40-69\% stenosed ICAs (Ackroyd et al., 1986), $310 \pm 99 \mathrm{~mL} / \mathrm{min}$ (mean \pm SD) for 50-75\% stenosed ICAs (Likittanasombut et al., 2006), and 167-399 $\mathrm{mL} / \mathrm{min}$ (with a peak of $526 \mathrm{~mL} / \mathrm{min}$ ) for ICA (Lindegaard et al., 1987).

Our CFD analysis showed that the peak velocities in CCA and ICA were 0.94 and $1.21 \mathrm{~m} / \mathrm{s}$, respectively, which fall within the ranges of the peak systolic velocity measured invasively as $0.47-1.14 \mathrm{~m} / \mathrm{s}$ in CCA and $0.94-3.05 \mathrm{~m} / \mathrm{s}$ in ICA for $60-90 \%$ stenotic carotid arteries (Blackshear et al., 1980). The pressure drop in our CFD analysis was also similar to the results of the patient-specific fluid-solid interaction (FSI) analyses by Tao et al. (2015).

The values of wall shear stress were reported to be $0.014 \mathrm{kPa}$ (peak shear stress at the plaque surface by FSI analyses for patient-specific CAB) (Kock et al., 2008) and $0.093 \mathrm{kPa}$ (mean fluid shear stress by 3D FSI analyses for atherosclerotic $\mathrm{CAB}$ with ruptured plaque) (Teng et al., 2010a). These values are much lower than the systolic pressure of $16 \mathrm{kPa}$, indicating that the longitudinal shear stress is too small to deform the fibrous cap.

\subsection{Limitations}

The extent of the lumenward deformation of the fibrous cap may be affected by the pulsatile blood flow conditions, which were not incorporated into the analyses in this study. A CFD analysis of the deformed shape of CAB under physiological loading, which also remains to be conducted, would provide more accuracy in terms of the pressure conditions on the vascular wall. Furthermore, region-dependent material properties, such as of the stiff fibrous cap (Holzapfel et al., 2014) and the residual stress/strain (Ohayon et al., 2007; Holzapfel et al., 2014), were not incorporated into the analyses here, but should be focused on in future studies. Another limitation is that the unruptured models were made by using the geometry of a ruptured carotid artery without considering remodeling. This caused some errors in predicting the deformation and stress/strain states for unruptured plaques by the FE method. We also ignored surrounding tissues such as perivascular adipose tissue (Brown et al., 2014). Although the adipose tissue was reported to be very soft (Comley and Fleck, 2010), further study is required to determine the resistive effect on vascular deformation.

\section{Conclusions}

We investigated the deformation of a ruptured plaque with IPH by FE analysis and compared it with soft and hard unruptured plaques. The rupture-induced pressure in the lipid core deformed the plaque lumenward, while the soft plaque in the unruptured model deformed outward and had a lumen with a round shape. The small area of the lumen in the ruptured model was also obtained in a hard plaque model with no IPH.

Our results indicate that geometrical change of a ruptured plaque with IPH is caused by an increase of rupture-induced pressure in the lipid core cavity, which may increase stenosis. A better understanding of the geometrical characteristics of plaques with IPH on contrast-enhanced ultrasonographic images may facilitate the identification of rupture.

\section{Acknowledgments}

This study was supported by JSPS KAKENHI (grant number 26420018). The authors thank Ms. Hiroyo Fukagawa for her technical assistance with the histological observations.

\section{Appendix}




\section{A1. RNG $k$ - $\varepsilon$ turbulence model}

The $k-\varepsilon$ transport equations are expressed as (Abaqus 6.14)

$$
\frac{d}{d t} \int_{V} \rho k d V+\int_{S} \rho k\left(v-v_{m}\right) \cdot n d S=\int_{S} \frac{\left(\mu+\mu_{T}\right)}{\sigma_{k}} \nabla k \cdot n d S+\int_{V} \tau_{i j} S_{i j} d V-\int_{V} \rho \varepsilon d V,
$$

and

$$
\frac{d}{d t} \int_{V} \rho \varepsilon d V+\int_{S} \rho \varepsilon\left(v-v_{m}\right) \cdot n d S=\int_{S} \frac{\left(\mu+\mu_{T}\right)}{\sigma_{\varepsilon}} \nabla \varepsilon \cdot n d S+\int_{V} C_{\varepsilon 1} \frac{\varepsilon}{k} \tau_{i j} S_{i j} d V-\int_{V} C_{\varepsilon 2} \frac{\rho \varepsilon^{2}}{k} d V .
$$

The second and third terms on the right hand side of the above equations represent the production and dissipation of $k$ and $\varepsilon$, respectively. The symbol $V$ denotes an arbitrary control volume surrounded by a surface area $S$, and variables $\boldsymbol{n}, \boldsymbol{v}, \boldsymbol{v}_{m}$, and $\rho$ are an outward unit vector normal to a surface $d S$, a velocity vector, velocity vector of a moving mesh, and fluid density, respectively.

The relationship between the Reynolds stress tensor $\tau_{i j}$ and the strain rate tensor $S_{i j}$ is expressed as

$$
\tau_{i j}=2 \mu_{T} S_{i j}=\mu_{T}\left(\frac{\partial u_{i}}{\partial x_{j}}+\frac{\partial u_{j}}{\partial x_{i}}\right)
$$

where the turbulent viscosity $\mu_{T}$ is defined as

$$
\mu_{T}=\frac{\rho C_{\mu} k^{2}}{\varepsilon} \text {. }
$$

The variable $C_{\varepsilon_{2}}$ is defined as

$$
C_{\varepsilon 2}=\tilde{C}_{\varepsilon 2}+\frac{C_{\mu} \eta^{3}\left(1-\frac{\eta}{\eta_{0}}\right) k^{2}}{1+\beta \eta^{3}}
$$

where

$$
\eta=\frac{k}{\varepsilon} \sqrt{2 S_{i j} S_{i j}}
$$

The default values of the parameters $C_{\mu}, C_{\varepsilon 1}, \tilde{C}_{\varepsilon 2}, \sigma_{\varepsilon}, \sigma_{k}, \beta$, and $\eta_{0}$, namely, $0.085,1.42,1.68,0.72,0.72$, 0.012, and 4.38, respectively, were used for the CFD analysis in Abaqus.

\section{A2. Inlet conditions and initial conditions}

Assuming that the artery is a smooth duct (Banks and Bressloff, 2007), the inlet turbulent flow intensity $I$ was estimated by

$$
I=0.16\left(\operatorname{Re}_{D_{H}}\right)^{-1 / 8}
$$

The variable $\operatorname{Re}_{D_{H}}$ is the Reynolds number of the inlet flow, which was derived as

$$
\operatorname{Re}_{D_{H}}=\frac{\rho U D_{C C A}}{\mu}
$$

where $\mu, U$, and $D_{C C A}$ are the dynamic viscosity, average inlet velocity, and the inner diameter of the wall (CCA side), respectively. Using the peak flow rate of $1100 \mathrm{~mL} / \mathrm{min}$ for a stenosed carotid bifurcation (Lee et al., 2008), the inlet velocity $U$ was calculated as $0.588 \mathrm{~m} / \mathrm{s}$ at the proximal side of the CCA.

Initial values for $k$ and $\varepsilon$ can be estimated by

$$
\begin{aligned}
& k=\frac{3}{2}(U I)^{2} \\
& \varepsilon=\frac{C_{\mu}^{3 / 4} k^{3 / 2}}{l},
\end{aligned}
$$

where $l$, the turbulent length scale, can be estimated by

$$
l=0.07 D_{C C A} \text {. }
$$




\section{References}

Abaqus 6.14, Abaqus analysis user's guide, SIMULIA, pp 6.6.2-10-6.6.2-15.

Ackroyd, N., Gill, R., Griffiths, K., Kossoff, G. and Appleberg, M., Quantitative common carotid artery blood flow: prediction of internal carotid artery stenosis, Journal of Vascular Surgery, Vol.3, No.6 (1986), pp. 846-853.

Banks, J. and Bressloff, N. W., Turbulence modeling in three-dimensional stenosed arterial bifurcations, Journal of Biomechanical Engineering, Vol.129, No.1 (2007), pp.40-50.

Blackshear, W. M., Phillips, D. J., Chikos, P. M., Harley, J. D., Thiele, B. L. and Strandness Jr., D.E., Carotid artery velocity patterns in normal and stenotic vessels, Stroke, Vol.11, No.1 (1980), pp.67-71.

Brown, N. K., Zhou, Z., Zhang, J., Zeng, R., Wu, J., Eitzman, D. T., Chen, Y. E. and Chang, L., Perivascular adipose tissue in vascular function and disease: a review of current research and animal models, Arteriosclerosis, Thrombosis, and Vascular Biology, Vol.34 (2014), pp.1621-1630.

Cai, J., Hatsukami, T. S., Ferguson, M. S., Kerwin, W. S., Saam, T., Chu, B., Takaya, N., Polissar, N. L. and Yuan, C., In vivo quantitative measurement of intact fibrous cap and lipid-rich necrotic core size in atherosclerotic carotid plaque: comparison of high-resolution, contrast-enhanced magnetic resonance imaging and histology, Circulation, Vol.112, No.22 (2005), pp.3437-3444.

Cardoso, L. and Weinbaum, S., Changing views of the biomechanics of vulnerable plaque rupture: a review, Annals of Biomedical Engineering, Vol.42, No.2 (2014), pp.415-431.

Chu, B., Kampschulte, A., Ferguson, M. S., Kerwin, W. S., Yarnykh, V. L., O’Brien, K. D., Polissar, N. L., Hatsukami, T. S. and Yuan, C., Hemorrhage in the atherosclerotic carotid plaque: a high-resolution MRI study, Stroke, Vol.35, No.5 (2004), pp.1079-1084.

Comley, K. and Fleck, N. A., A micromechanical model for the Young's modulus of adipose tissue, International Journal of Solids and Structures, Vol.47 (2010), pp.2982-2990.

Davies, M. J., Pathology of arterial thrombosis, British Medical Bulletin, Vol.50, No.4 (1994), pp.789-802.

Delfino, A., Stergiopulos, N., Moore Jr., J. E. and Meister, J. J., Residual strain effects on the stress field in a thick wall finite element model of the human carotid bifurcation, Journal of Biomechanics, Vol.30 (1997), pp.777-986.

European Carotid Surgery Trialists' Collaborative Group, MRC European carotid surgery trial: interim results for symptomatic patients with severe (70-99\%) or with mild (0-29\%) carotid stenosis, Lancet, Vol.337, No.8752 (1991), pp.1235-1243

Groen, H. C., Gijsen, F. J., van der Lugt, A., Ferguson, M. S., Hatsukami, T. S., van der Steen, A. F., Yuan, C. and Wentzel, J. J., Plaque rupture in the carotid artery is localized at the high shear stress region: a case report, Stroke, Vol.38, No.8 (2007), pp.2379-81.

Hamada, O., Sakata, N., Ogata, T., Shimada, H. and Inoue, T., Contrast-enhanced ultrasonography for detecting histological carotid plaque rupture: quantitative analysis of ulcer, International Journal of Stroke, Vol.11, No.7 (2016), pp.791-798.

Hennerici, M. G., The unstable plaque, Cerebrovascular Diseases, Vol. 17, Supplement 3 (2004), pp. 17-22.

Holzapfel, G. A., Mulvihill, J. J., Cunnane, E. M. and Walsh, M. T., Computational approaches for analyzing the mechanics of atherosclerotic plaques: a review, Journal of Biomechanics, Vol.47, No.4 (2014), pp.859-869.

Jackson, Z. S., Dajnowiec, D., Gotlieb, A. I. and Langille, B. L., Partial off-loading of longitudinal tension induces arterial tortuosity, Arteriosclerosis, Thrombosis, and Vascular Biology, Vol.25 (2005), pp.957-962.

Kock, S. A., Nygaard, J. V., Eldrup, N., Frund, E. T., Klaerke, A., Paaske, W. P., Falk, E. and Yong Kim, W., Mechanical stresses in carotid plaques using MRI-based fluid-structure interaction models, Journal of Biomechanics, Vol.41, No.8 (2008), pp.1651-1658.

Ku, D. N., Blood flow in arteries, Annual Review of Fluid Mechanics, Vol.29 (1997), pp.399-434.

Lee, S. E., Lee, S., Fischer, P.F., Bassiouny, H.S. and Loth, F., Direct numerical simulation of transitional flow in a stenosed carotid bifurcation, Journal of Biomechanics, Vol.41, No.11 (2008), pp.2551-2561.

Likittanasombut, P., Reynolds, P., Meads, D. and Tegeler, C., Volume flow rate of common carotid artery measured by doppler method and color velocity imaging quantification (CVI-Q), Journal of Neuroimaging, Vol.16, No.1 (2006), pp.34-38.

Lindegaard, K. F., Lundar, T., Wiberg, J., Sjøberg, D., Aaslid, R. and Nornes, H., Variations in middle cerebral artery blood flow investigated with noninvasive transcranial blood velocity measurements, Stroke, Vol.18, No.6 (1987), 
pp.1025-1030.

Lovett, J.K., Gallagher, P.J., Hands, L.J., Walton, J. and Rothwell, P.M., Histological correlates of carotid plaque surface morphology on lumen contrast imaging, Circulation, Vol.110, No.15 (2004), pp.2190-2197.

Ogata, T., Inoue, T. and Okada, Y., Outcome of 312 Japanese patients with carotid endarterectomy and factors associated with cardiovascular events-a single-center study in Japan, Journal of Stroke and Cerebrovascular Diseases, Vol.23, No.3 (2014), pp.529-33.

Ohayon, J., Dubreuil, O., Tracqui, P., Le Floc'h, S., Rioufol, G., Chalabreysse, L., Thivolet, F., Pettigrew, R. I. and Finet, G., Influence of residual stress/strain on the biomechanical stability of vulnerable coronary plaques: potential impact for evaluating the risk of plaque rupture, American Journal of Physiology-Heart and Circulatory Physiology, Vol.293, No.3 (2007), pp.H1987-1996.

Pasterkamp, G. and van der Steen, A. F., Intraplaque hemorrhage: an imaging marker for atherosclerotic plaque destabilization? Arteriosclerosis, Thrombosis, and Vascular Biology, Vol.32, No.2 (2012), pp.167-168.

Rothwell, P. M., Gibson, R. and Warlow, C.P., Interrelation between plaque surface morphology and degree of stenosis on carotid angiograms and the risk of ischemic stroke in patients with symptomatic carotid stenosis, Stroke, Vol.31, No.3 (2000), pp.615-621.

Saam T, Ferguson M, Yarnykh V, Takaya N, Xu D, Polissar N, Hatsukami T, Yuan C Quantitative evaluation of carotid plaque composition by in vivo MRI. Arterioscler Thromb Vasc Biol 25(1) (2005) pp.234-239

Saam, T., Hetterich, H., Hoffmann, V., Yuan, C., Dichgans, M., Poppert, H., Koeppel, T., Hoffmann, U., Reiser, M. F. and Bamberg, F., Meta-analysis and systematic review of the predictive value of carotid plaque hemorrhage on cerebrovascular events by magnetic resonance imaging, Journal of American College of Cardiology, Vol.62, No.12 (2013), pp.1081-1091.

Sommer, G., Regitnig, P., Koltringer, L. and Holzapfel, G. A., Biaxial mechanical properties of intact and layer-dissected human carotid arteries at physiological and supraphysiological loadings, American Journal of Physioly-Heart and Circulatory Physiology, Vol.298, No.3 (2010), pp.H898-H912.

Stary, H. C., Chandler, A. B., Dinsmore, R. E., Fuster, V., Glagov, S., Insull, W., Rosenfeld, M. E., Schwartz, C. J., Wagner, W. D. and Wissler, R. W., A definition of advanced types of atherosclerotic lesions and a histological classification of atherosclerosis: a report from the Committee on Vascular Lesions of the Council on Arteriosclerosis, American Heart Association, Arteriosclerosis, Thrombosis, and Vascular Biology, Vol.15, No.9 (1995), pp.1512-1531.

Takano, K., Yamashita, S., Takemoto, K., Inoue, T., Sakata, N., Kuwabara, Y. and Yoshimitsu, K., Characterization of carotid atherosclerosis with black-blood plaque imaging using variable flip-angle 3D turbo spin-echo: comparison with 2D turbo spin-echo sequences, European Journal of Radiology, Vol.81, No.3 (2012), pp.E304-E309.

Tan, F. P., Soloperto, G., Bashford, S., Wood, N. B., Thom, S., Hughes, A. and Xu, X. Y., Analysis of flow disturbance in a stenosed carotid artery bifurcation using two-equation transitional and turbulence models, Journal of Biomechanical Engineering, Vol.130, No.6 (2008), p.061008.

Tao, X., Gao, P., Jing, L., Lin, Y. and Sui, B., Subject-specific fully-coupled and one-way fluid-structure interaction models for modeling of carotid atherosclerotic plaques in humans, Medical Science Monitor, Vol.21 (2015), pp.3279-3290.

Teng, Z., Canton, G., Yuan, C., Ferguson, M., Yang, C., Huang, X., Zheng, J. and Woodard, P. K. Z., 3D Critical plaque wall stress is a better predictor of carotid plaque rupture sites than flow shear stress: an in vivo MRI-based 3D FSI study, Journal of Biomechanical Engineering, Vol.132, No.3 (2010a), p.031007-1.

Teng, Z., Sadat, U., Li, Z., Huang, X., Zhu, C., Young, V. E., Graves, M. J. and Gillard, J. H., Arterial luminal curvature and fibrous-cap thickness affect critical stress conditions within atherosclerotic plaque: an in vivo MRI-based 2D finite-element study, Annals of Biomedical Engineering, Vol.38, No.10 (2010b), pp.3096-3101.

Yakhot, V., Orszag, S. A., Thangam, S., Gatski, T. B. and Speziale, C. G., Development of turbulence models for shear flows by a double expansion technique, Physics of Fluids, Vol.4, No.7 (1992), pp.1510-1520.

Yamada, H., Yuri, K. and Sakata, N., Correlation between stress/strain and the retention of lipoproteins and rupture in atheromatous plaque of the human carotid artery: a finite element study, Journal of Biomechanical Science and Engineering, Vol.5, No.4 (2010), pp.291-302.

Yamada, H. and Sakata, N., Low pressure condition of a lipid core in an eccentrically developed carotid atheromatous plaque: a static finite element analysis, Journal of Biorheology, Vol.27, No.1 (2013), pp.9-17. 